\title{
Therapeutic Containment Protocol in the Management of Harm Risk Situation: A Practical Proposal for a Staggered Clinical Procedure
}

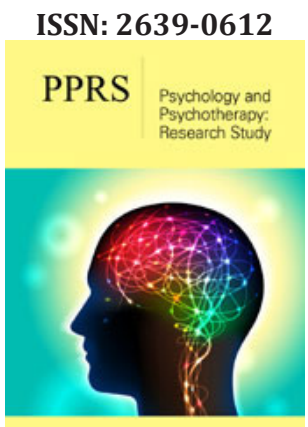

*Corresponding author: Jesús Alejandro $\mathrm{AL}$, Teaching Department, Center for comprehensive mental health care extended stay, Mexico

Submission: 眥 August 10, 2019

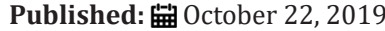

Volume 2 - Issue 5

How to cite this article: Jesús Alejandro AL, Rafael MD, Jorge SR, Tomás HBD, Martín PA. Therapeutic Containment Protocol in the Management of Harm Risk Situation: A Practical Proposal for a Staggered Clinical Procedure. Psychol Psychother Res Stud. 2(5).PPRS.000547.2019.

DOI: 10.31031/PPRS.2019.02.000547

Copyright@ Jesús Alejandro AL, This article is distributed under the terms of the Creative Commons Attribution 4.0 International License, which permits unrestricted use and redistribution provided that the original author and source are credited.

\author{
Jesús Alejandro $\mathrm{AL}^{1 *}$, Rafael $\mathrm{MD}^{2,3}$, Jorge $\mathrm{SR}^{2}$, Tomás $\mathrm{HBD}^{2}$ and Martín $\mathrm{PA}^{4}$ \\ ${ }^{1}$ Teaching department, Mexico \\ ${ }^{2}$ Comprehensive mental healthcare center-Prolonged Staying, Mexico \\ ${ }^{3}$ Clinic department of health sciences Center, Mexico \\ ${ }^{4}$ Mental health nurse, Mexico
}

\begin{abstract}
Physical restraint and seclusion have been well-known practices in medicine, and particularly in psychiatric settings worldwide. These controversial interventions should lead to Mental healthcare facilities to have a defined strategy to safely and effectively manage patients in high harm risk situations for him/herself and/or others, while ensuring the patients Human Rights, the United Nations Mental Illness Principles, and the torture prevention policies, aligned in a continuous patient's safety and quality improvement culture of mental healthcare. This paper provides a practical succinct Harm Risk Situation management protocol applicable for most healthcare services, emphasizing the therapeutic aim of the sequenced staggered procedures empowering the verbal containment method, and strictly leaving involuntary pharmacological and physical containment as the last resource available, for the shortest time possible, and under constant qualified supervision.
\end{abstract}

Keywords: Agitation; Aggression; Restraint; Verbal de-escalation; Containment; Harm risk; Human rights

Abbreviations: HRS: Harm Risk Situations; MHF: Mental Health Facility; MI: Mental Illness; CT: Containment Team

\section{Introduction}

Improving the safety and quality of healthcare services is an essential part of healthcare provider organizations, but regarding mental healthcare, there is scarce information mainly because the lack of standardized information technology-based data sources, and limited scientific evidence for mental health quality measures [1]. Because of the unique patient safety issues in mental healthcare, related to clinical harm risk situations (HRS), like aggression, violence, suicide or self-harm [2], and particularly for the strategies to manage them, like physical restraint, it is much needed to develop protocoled collaborative procedures, in order to ensure not only the safety of the patient and others, but the dignification of his/her human rights. An extensive controversy about the safety and ethical considerations in the practice of physical restraint in medicine, but particularly in psychiatry, has prompted in 1991 the promulgation of the United Nations Principles for the Protection of Persons with Mental Illness and the Improvement of Mental Health Care (MI Principles), in which principle 11(11) stays that "Physical restraint or involuntary seclusion of a patient shall not be employed except in accordance with the officially approved procedures of the mental health facility (MHF) and only when it is the only means available to prevent immediate or imminent harm to the patient or others [3]. This statement is intended to discourage the use of restraints and seclusion in MHF in order to protect against abuse, limiting this procedure as a last and exceptional measure, addressed further in 2005 in the WHO Resource Book on Mental Health, Human Rights and Legislation, which details specific aspects in the application of the MI Principles [4]. Despite of these actions and the recognition of the deleterious effects of being physically restrained, as disruption of the therapeutic relationship [5], its association with longer length of stay, increased medication consumption and higher costs consequences [6], its application is still required, well documented, and widely used worldwide [7], especially in the emergency settings [8], but it may be poorly coordinated, supervised and staff training insufficient [9]. 
While the cochrane systematic review did not find randomized controlled studies in the matter [10], several guidelines have been made to address aggression and agitation [11], emphasizing the need of a dedicated protocol for specific procedures in the recognition and management of HRS, with appropriate and continuously training sessions for all the nursing and medical staff. In this paper, while not intended to be an exhaustive review of literature, but a well sustained directive practical proposal, we provide a succinct comprehensive structured protocol for the management of HRS, elaborated as part of the continuous improving health care quality and safety plan on our unit, that could also be applicable for most MHF, emergency rooms, inpatient care, and in some extent to community health services, conformed as a general stratified algorithm based in several guidelines recommendations, with ponderation in patient's human rights, and continuous quality improvement in the safety of patients and others, leaving the physical contention as the last option. It is resumed the general verbal de-escalation valuable principles, and the pharmacological approach to agitation. Also, the author's physical contention technique is described, along with specific nursing care management of a patient under physical containment.

\section{Discussion}

While physical restraint concept refers to limiting the freedom of movement, it is frequently associated as a form of coercive measure [12]. Similarly, chemical restraint is related to forced drug administration [13], acquiring a pejorative sense without reflecting any therapeutic purpose, even if it is restricted to limiting the harm. Otherwise, the term "containment" offers a more neutral and broad meaning, referring to the staff actions for the management of HRS [14], that could facilitate the communication with the patient [15], avoiding as possible the word "restraints", since it could be interpreted as coercive, increasing distrust and fear in the patient, with possible aggressive response escalation. Considered a form of torture, it is important to state that any coercive and non-consensual measures should be banned in all places of deprivation of liberty, including psychiatric and social care institutions [16], and that the severity of the mental illness is not by itself sufficient to justify detention, the state needs to show that detention is necessary to protect the safety of the person or of others. Seclusion is the involuntary confinement of a patient alone in a room or area from which is physically prevented from leaving [17]. We do not recommend seclusion as officially stated by the UN Special Rapporteur as a cruel, inhuman and degrading form of treatment on persons with mental disabilities [16], also finding it unpractical and complicated to install, since a special room or area requires protective walls and equipped doors to reduce the stimuli [18], which will not always be available in MHFs, and does not ensure the safety of the patient. This protocol will always recommend staggered interventions, starting from opportune prevention and identification of potential HRS, providing and maintaining the verbal containment recommendations during the entire management of HRS, with great emphasis in non-coercive collaborative approach, leaving the physical containment as the last resource, with the pharmacological containment resources in the middle, avoiding as possible involuntary medication.

\section{Identification of HRS}

Table 1: Basic features to consider in the clinic evaluation of harm risk situations [23].

\begin{tabular}{|c|c|c|c|}
\hline Components & Self-Harm/Suicide Risk & $\begin{array}{l}\text { Aggression/Violence (Harm Risk to } \\
\text { Others) }\end{array}$ & Mental Instability \\
\hline History & $\begin{array}{l}\text { History of self-harm behav- } \\
\text { ior } \\
\text { Previous suicidal attempts } \\
\text { Family suicide history } \\
\text { Physical/Sexual abuse } \\
\text { history }\end{array}$ & $\begin{array}{l}\text { Violence history } \\
\text { Conduct disorder history } \\
\text { No adherence history }\end{array}$ & $\begin{array}{l}\text { Poverty/Indigence } \\
\text { Poor or null family } \\
\text { support } \\
\text { Stigmatizing experiences }\end{array}$ \\
\hline Clinic & $\begin{array}{l}\text { Mental illness history } \\
\text { Severe medical condition } \\
\text { history } \\
\text { Alcohol or drugs use disor- } \\
\text { ders } \\
\text { Low frustration tolerance } \\
\text { history } \\
\text { Social isolation }\end{array}$ & $\begin{array}{l}\text { Command auditory halluci- } \\
\text { nations are of particular risk } \\
\text { Manic episode of Bipolar } \\
\text { Disorder } \\
\text { Impulse Control Disorder } \\
\text { Alcohol or drug use problems }\end{array}$ & $\begin{array}{l}\text { No adherence history } \\
\text { Poor self-grooming or } \\
\text { refuse to hygiene } \\
\text { Increased alcohol or } \\
\text { drugs consumption } \\
\text { Evidence of severe } \\
\text { symptomatology or } \\
\text { threating behavior }\end{array}$ \\
\hline
\end{tabular}




\begin{tabular}{|c|c|c|c|}
\hline Attitude & $\begin{array}{l}\text { Lack of emotional regulation } \\
\text { skills } \\
\text { Impulsiveness and emotional } \\
\text { labiality } \\
\text { Limited and counterproduc- } \\
\text { tive believes and behavior }\end{array}$ & $\begin{array}{l}\text { Rage attacks and emotional } \\
\text { regulation deficits } \\
\text { Low frustration tolerance } \\
\text { Antisocial cognition, } \\
\text { thoughts and believes }\end{array}$ & $\begin{array}{l}\text { Poor cognitive, emotion- } \\
\text { al and conduct skills } \\
\text { Mayor change in } \\
\text { behavior (e.g. excessive } \\
\text { expenses, euphoria, will } \\
\text { making) }\end{array}$ \\
\hline Context/Environment & $\begin{array}{l}\text { Loss due to death or separation (cou- } \\
\text { ple, work, pet) } \\
\text { A significant change in lifestyle (e.g., } \\
\text { retirement, children leave home, finan- } \\
\text { cial problems) }\end{array}$ & $\begin{array}{l}\text { Unstable or deficient family } \\
\text { or social support } \\
\text { Ruptured interpersonal or } \\
\text { family relationships } \\
\text { Age: More associated with } \\
\text { youth } \\
\text { Sex: More associated with } \\
\text { men }\end{array}$ & $\begin{array}{l}\text { Change in live style } \\
\text { Loss of interpersonal } \\
\text { relationships or job } \\
\text { Grief }\end{array}$ \\
\hline
\end{tabular}

Mental health services must have risk management systems and processes in place, implying the systematic risk identification, assessment and management. Aggressive behavior may escalate in a predictable and orderly manner, providing opportunities for the healthcare professional to assess and intervene [14]. HRS could be so explicit that it is easily recognized by most people, but the development of observational awareness in the services is essential to opportunely identify subtle and evident signs of potential HRS, this include psychomotor restlessness (pacing, repeatedly asking to see the doctor, slamming doors), voice volume increasing, verbal threats [19]. The HRS could be identified by any of the healthcare staff, either by direct observation, or by the report of a family member or other persons. Once identified, the nurse of the area should be notified, and goes where the patient is, performing intervention using verbal containment method, while assessing the elements of the current situation. HRSs could be differentiated into three general distinctive scenarios: "Self-harm/ Suicide risk", "Aggression/Violence (Harm risk to others)", and "Mental instability", each one with particular features that could be assessed by any clinical staff (Table 1); [20,21]. This first approach triggers the protocol algorithm in a sequenced fashion, performing a series of interventions depending on the evaluations of the HRS, and the type of response to interventions (Figure 1). Environmental modifications may be useful to prevent escalation of symptoms, like ensure physical comfort, reduce external stimuli (irritating light, noise, or ambient temperature), removal of objects that may be potentially dangerous, and maintenance of an optimal safe distance to respect the patient's personal space [22].

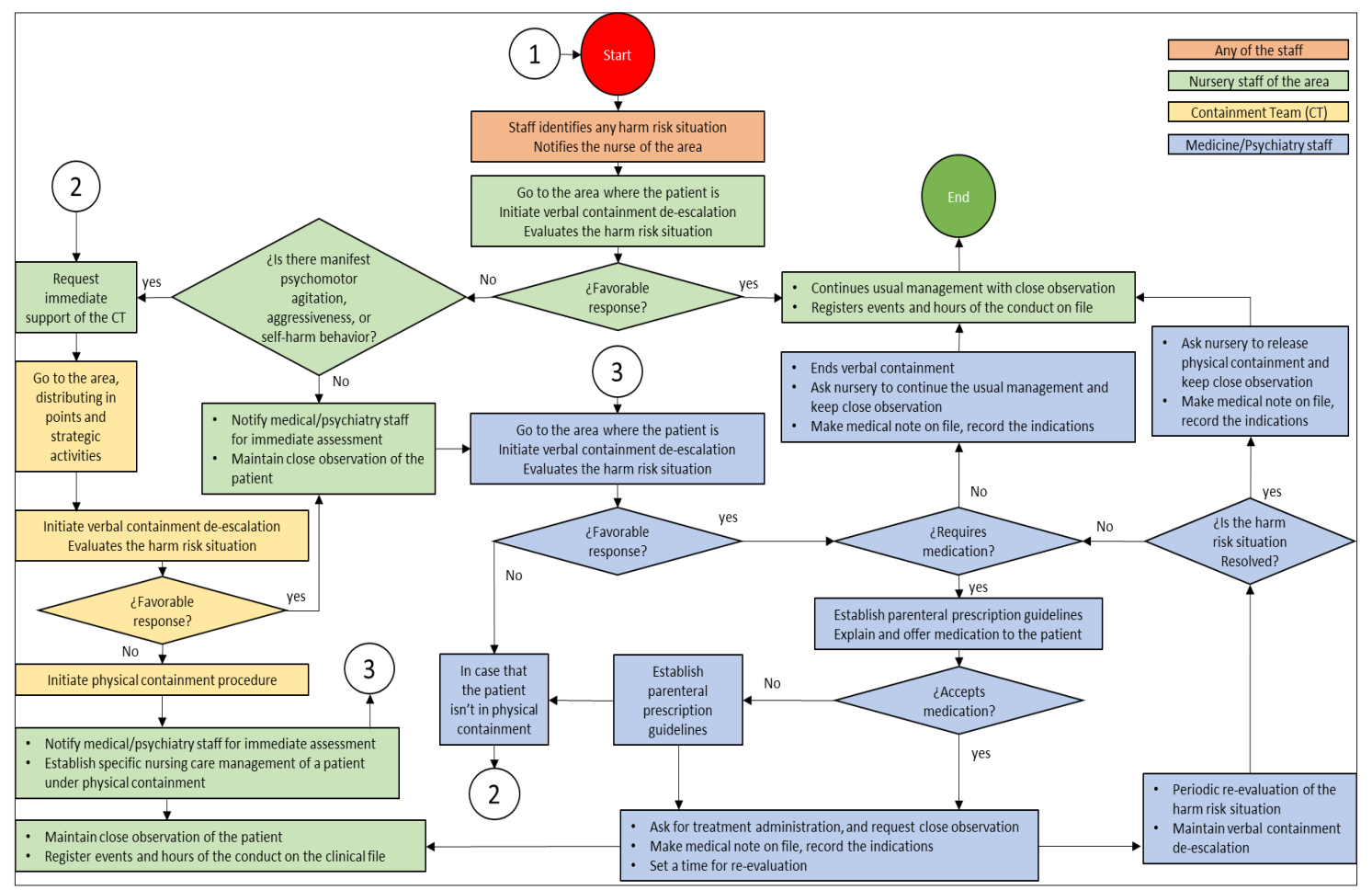

Figure 1: Therapeutic containment protocol algorithm. 


\section{Verbal containment}

Also referred as verbal de-escalation, this method has found successful outcomes improving staff relationships with patients and reducing the frequency of conflict and physical containment [15]. The aim is to help the patient to calm him/herself, allowing the patient to find his/her control centers. In this strategy, the clinician listens to the patient and finds a way to respond that agrees with or validates the patient's position, then states a recommendation (e.g., take medication, sit down, etc.). This loop repeats to the patient's response, based on the 10 commandments proposed by Fishkind [19]:

i. You shall respect personal space: 2-times-arm-length rule (twice your arm length) is an appropriate distance that's generally accepted as non-threatening, but the patient may want you to increase your distance. Maintain your usual social eye contact, avoid staring or a direct gaze that could be interpreted as aggressive behavior. Procure always an "escape route" for you and the patient, don't make the patient feel he/she is trapped.

ii. You shall not be provocative: A calm demeanor and facial expression are important. Be soft-spoken, use relaxed stance with your knees bent, arms uncrossed, hands in sight, and the palms upward. Never threaten the patient, the initial therapeutic alliance is a critical factor in effective verbal containment.

iii. You shall establish verbal contact: Ask and learn the patient's name and address him or her using the last name, as using the first name may be perceived as too personal or not genuine. Identify yourself, tell the patient who you are, and establish that your job is to keep the patient safe and to allow no harm to befall him/her, offer reassurance that you want to help him or her regain control.

iv. You shall be concise and repeat yourself: ("Less is more"), use short phrases with a simple vocabulary, wordiness will cause confusion. Repetition is essential, as the patient may not hear you the first time, whenever you set limits, offer choices or propose alternatives.

v. You shall identify wants and feelings: Once gotten the patient's attention, empathize and solidify the therapeutic alliance recognizing the patient's wants and feelings (e.g. "You seem angry; is there something you want that you're not getting? Perhaps I can get it for you."). Also explore and ask this wants and feelings.

vi. You shall listen: Try to understand what the patient is saying (not what you think he/she is saying). I find it helpful to make sure that you have correctly understood by commenting, "Let me see if I understand you correctly". Don't argue with the patient.

vii. You shall agree or agree to disagree: Agreeing with a patient without furthering a delusion or lying, could be very difficult, but is a useful way to validate the patient's experience (e.g. The patient thinks aliens are torturing him, agree by saying "I have not seen the aliens or seen you tortured, but I believe you are being tortured"). This could diffuse the patient's anger. Agree for as long as you can with the patient's experience. If you cannot go any further, you can always say, "We can agree to disagree."

viii. You shall lay down the law: Describe to the patient the acceptable behavior expected from him/her. Be honest telling the patient that his/her conduct is scaring you or other persons. Tell the patient that injury to him/herself or to others is unacceptable. Be prepared to be challenged repeatedly as you set firm limits, you may need to tell the patient there are consequences to the behavior.

ix. You shall offer choices: Some patients may believe there is nothing left but to fight or flight, offering choices such as taking a time-out, or a medication to decrease the anger, can be a welcome relief. If an assault is imminent, don't expect the patient to engage in problem solving, be assertive.

x. You shall debrief the patient and staff: Some patients will still end up in physical containment, some may require immediate intramuscular pharmacological containment, so it is recommended to debrief the patient once the HRS is over and the patient is calm. This could restore a therapeutic relationship, diminishing the traumatic nature of the events, and decrease the risk of additional violent events. In addition, individual and collective inpatient perspectives should inform alternatives to physical containment (Ling).

The amount of time permitted for verbal contention may vary depending on the setting and HRS type, however, can be successfully delivered in less than 5 minutes with some extra time needed for the complicated cases, usually taking 10 minutes at the most. When effectively delivered, verbal containment can avoid the need for physical contention. Taking time to de-escalate the patient, and work with him/her as he/she settles down can be much less timeconsuming than displaying physical containment that requires additional resources [23].

\section{Pharmacological containment}

The use of medication in HRS is a valuable resource to rapidly calm the patient as the primary goal, avoiding as far as possible the sedation of the patient. Some recommendations should be taken when pharmacological containment is contemplated: Using the medication too quickly may seem derogatory, reject or humiliate the patient, and may lead to further escalation, so offering this alternative should be aligned with the verbal containment principles. It is always preferred to seek oral administration route, also sublingual or dispersible medications are useful. Consider the side effects of each medication and drug interactions. Assess the speed of the route of administration. We recommend the Consensus Statement of the American Association for Emergency Psychiatry Project Protocol for treatment of agitation [24]; (Figure 2), always considering the potential causes of the condition, including acute intoxication, psychiatric illness, delirium, etc.

\section{Physical containment}

The following is a guide to perform a safe physical containment procedure developed by the authors Ramírez, Bolard and Padilla, 
based on several and practical wrestling immobilization techniques that aims limiting the movements of the patient, also preventing the patient's or team member's lesions. For the delivering of physical containment, it is required a minimum of 5 persons, 4 fastening belts, 4 presses, and 1 observation bed. In order ensure the safety in the complex dynamics of physical containment delivering, we state that the conformation of a high performance team is required, analog to the advanced cardiac life support resuscitation team, allowing systematic and highly organized set of assessments and treatments to take place simultaneously, in order to efficiently and effectively resolve a HRS as a Containment Team (CT). In order for this to happen, it requires a healthcare professionals' team highly trained on the mastery of verbal and physical containment, to act in an organized communicative and effective manner, understanding not only the individual role, but other team members role as well, helping each team member to anticipate what may be expected next and helping them perform their role with efficiency and safety:

a. One First Containment Professional: Will approach the patient, keeping a distance from 1 to 2 steps.

b. One Environment Monitoring Professional: Will be kept within 5 steps of the patient, supervising the activities and situation of the environment, also functioning as a protection barrier for other people. c. Two Strategic Exit-points Professionals: They will keep a distance of between 5 to 10 steps from the patient or standing outside of the consulting room, generating a security perimeter around the patient.

d. One Circulating Support Professional: Will clear the route of transfer the patient to the observation area, removing objects that may hinder it, or with harm potential, opens the doors that are necessary, prepares and sets the fastening material in the bed observational.

Once the decision that physical containment is necessary and not contra-indicated (Recent eye or brain surgery), staff prepares for the containment procedure, as the patient and/or their family should be informed and consent requested although non-consent by the patient does not preclude the application of physical restraint18.

\section{Physical containment procedure}

1. The First Containment Professional (Figure 3) identify him/herself with his name and badge to the patient (Figure 3), always maintaining verbal contact explaining the current situation and the procedure to be performed, and makes a notification to the Environment Monitoring Professional (Figure 3)

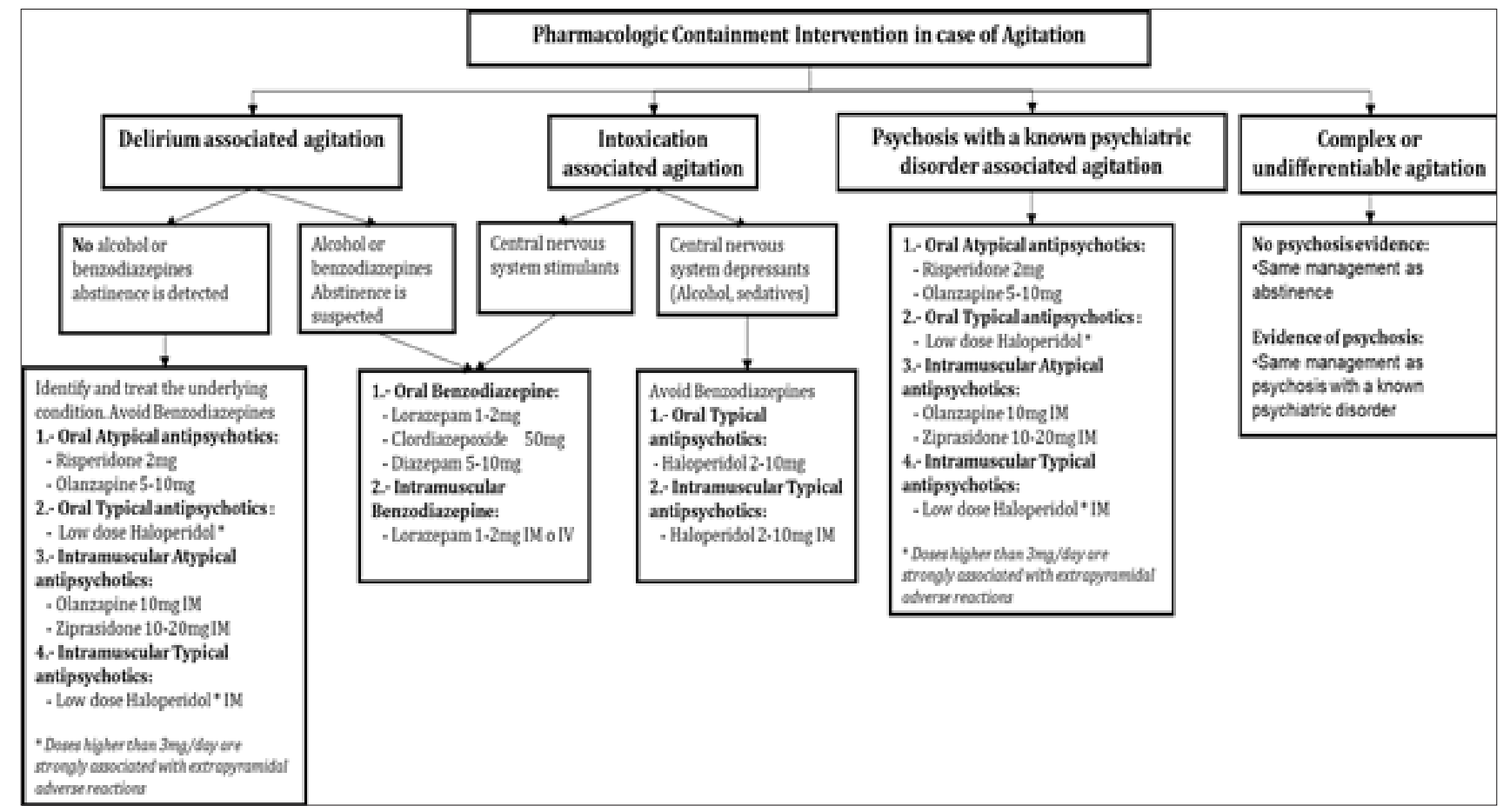

Figure 2: Pharmacological containment intervention in case of agitation [23].

2. The Environment Monitoring Professional (Figure 4) makes a notification signal to the Strategic Exit-points Professionals (Figure 4) to approach and support the management of the situation.

3. The Circulating Support Professional: Starts to clear the route to the observation area (Figure 5), removing objects that may hinder the procedure and that represent potential for risk of damage, notifies the staff of the area, prepares the material securing with the presses the straps of the fastening belts (Figure 6) to the frame of the intended bed which must be free of railings. 


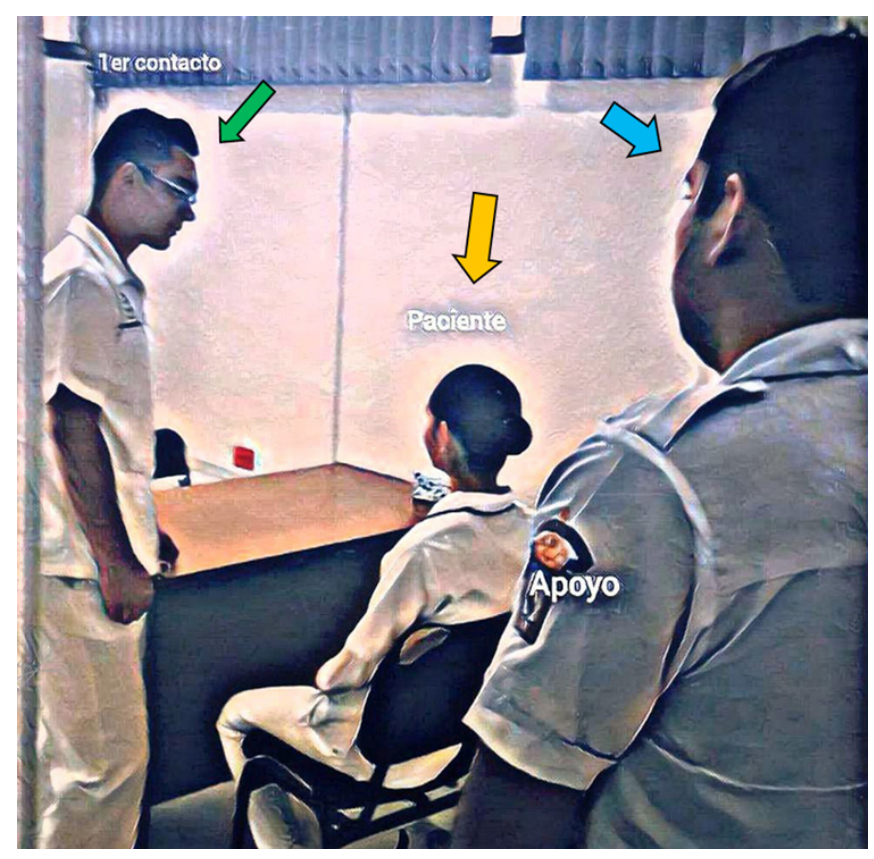

Figure 3:
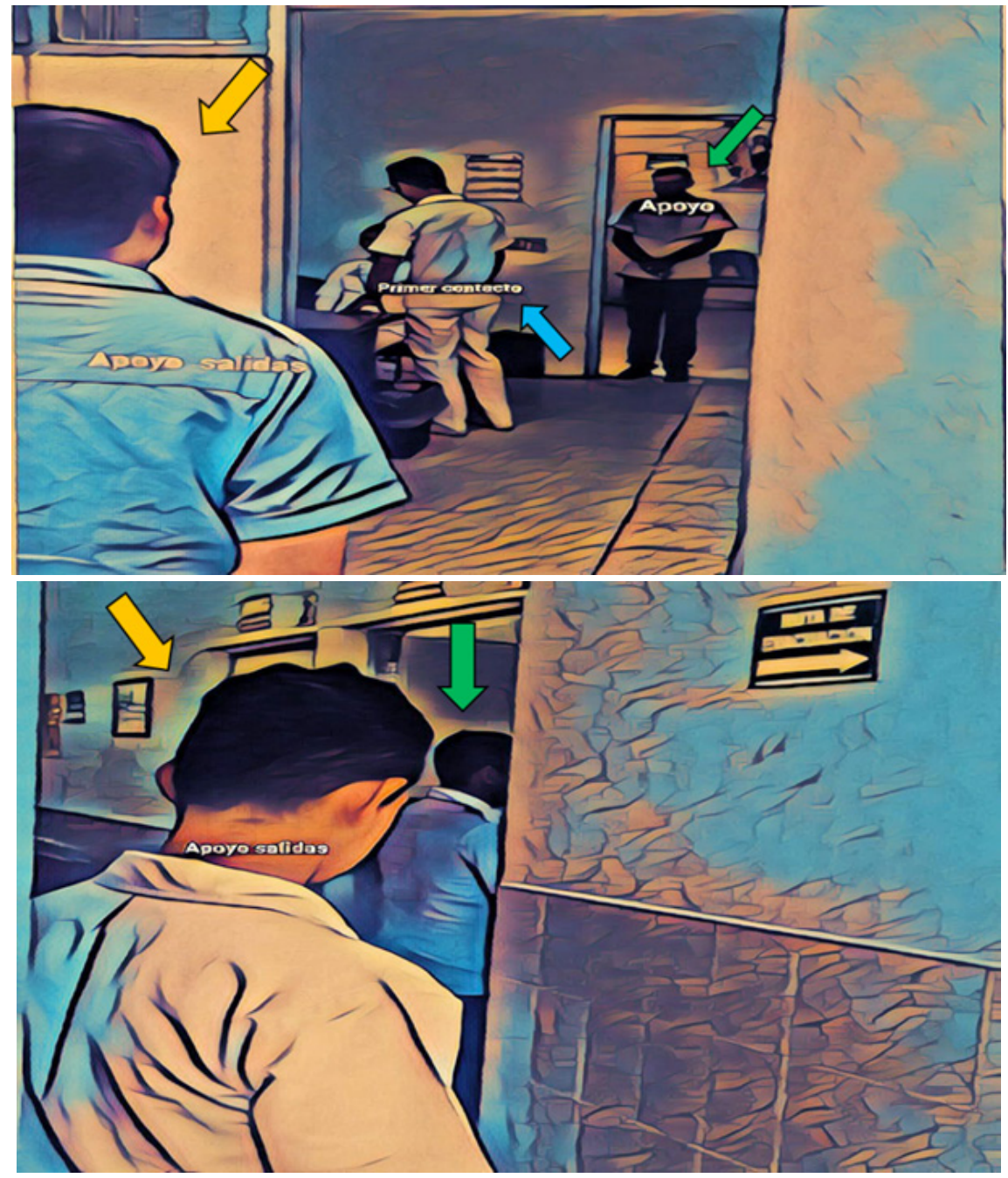

Figure 4: 


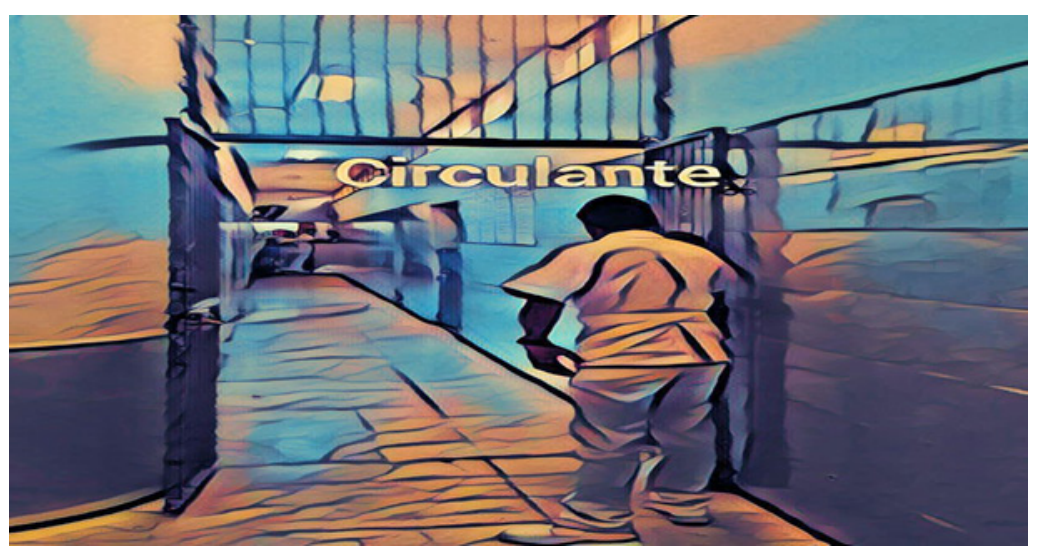

Figure 5:

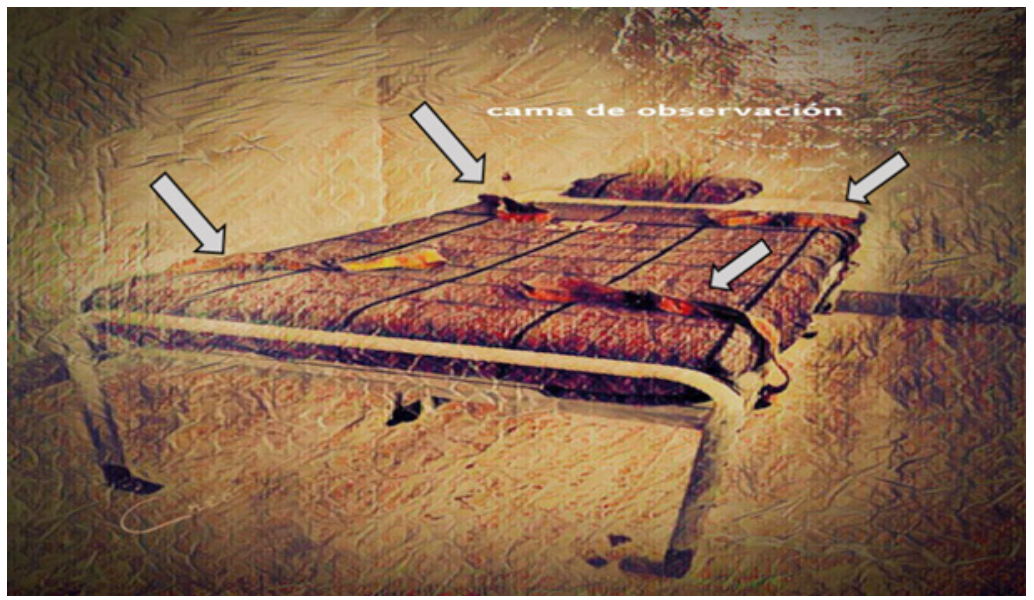

Figure 6:

4. The CT, proceeds to immobilize the forearms and professional's palm on the internal side of the patient's wrist, shoulders of the patient as follows:

a. The patient's wrist is gently pressed by placing the

leaving the professional's index, middle, annular and pinky fingers on the patient's ulnar wrist, while the professional's thumb places on the patient's radial wrist (Figure 7).

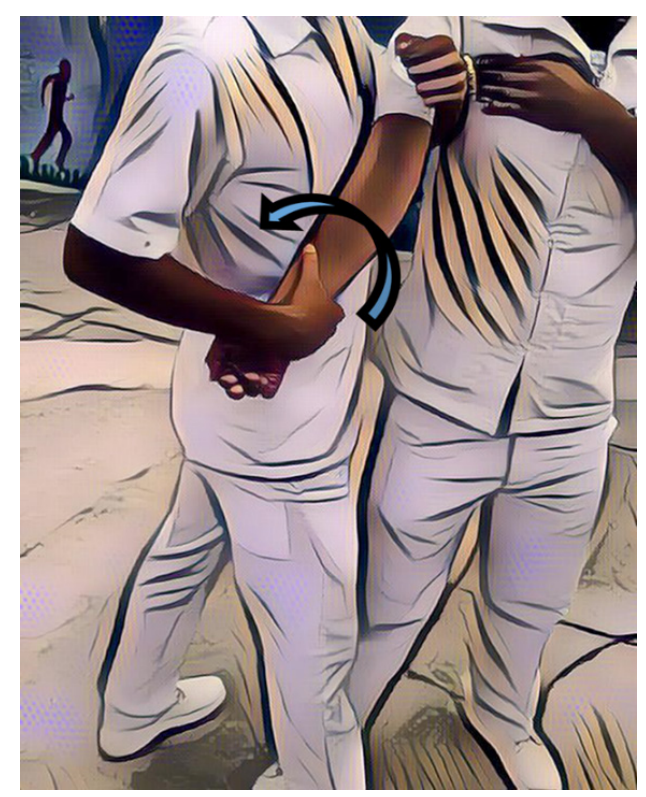

Figure 7: 
b. The patient's wrist is gently rotated outward in an abduction movement, to neutralize forearm and hand movements (Figure 7).

c. The professional's available arm slides between the patient's arm and thorax (Figure 8), positioning the professional's forearm in the patient's axillary hollow, leaving the professional's elbow facing the patient's back, providing a better control of the thoracic movements.

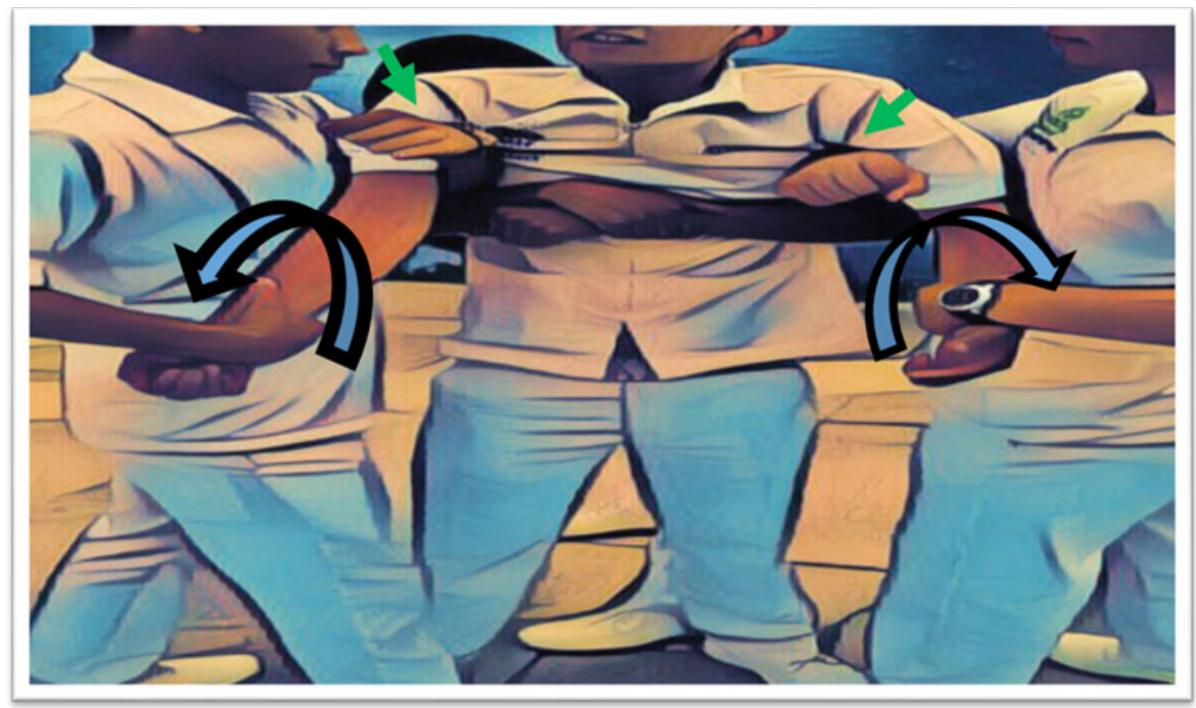

Figure 8:

d. The professional's foot is placed in front of the patient's foot, to stabilize and limit the lower movements (Figure 9).

e. One Strategic Point Professional simultaneously stabilize the chest movements, by hugging the patient from the back and joining their hands in the patient's sternum (Figure 9), lowering their head at the level and between patient's scapula's, to protect themselves from backward movements of patient's head (Figure $10)$.

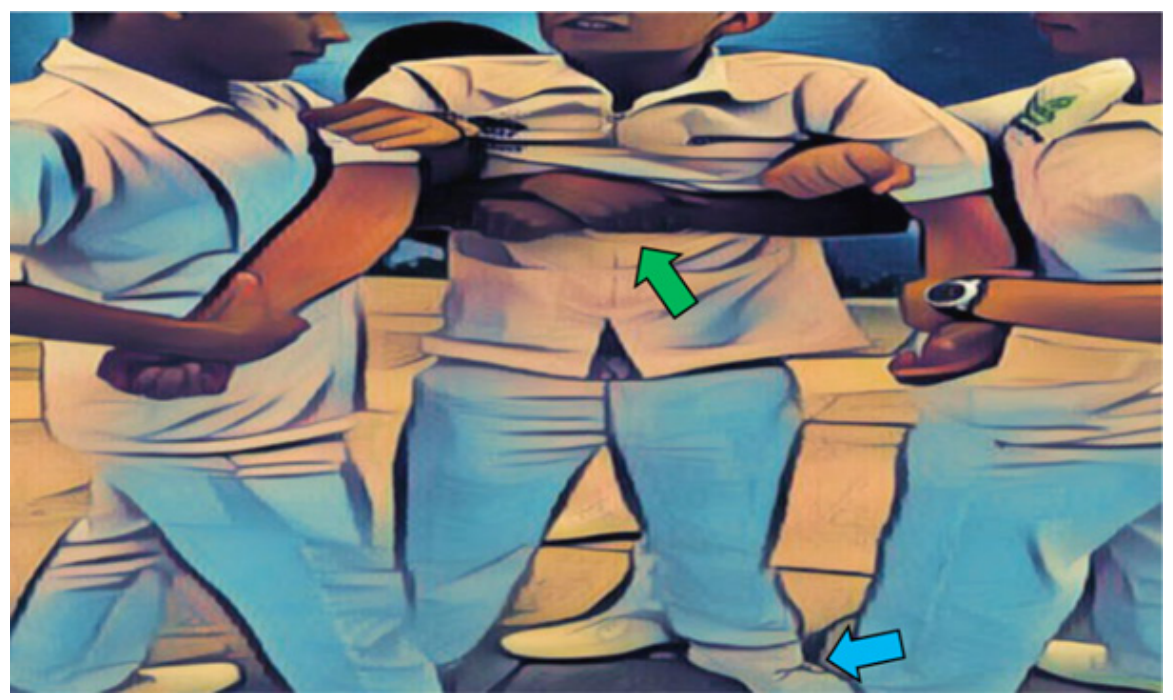

Figure 9:

5. Once the patient's movements have been successfully secured and limited, he/she will be escorted and assisted to be transferred to the area where the therapeutic support will be carried out, and his observation will be maintained.

6. Upon arrival at the area destined for therapeutic support, the patient is assisted to lie down the bed, where if uncooperative, the Circulating Professional and one Strategic Point Professional will proceed to immobilize lower limbs as follows:

a. The professional's forearm will slide from the inside side of the patient's knee (Figure 11) outwardly, leaving the professional's elbow behind the patient's popliteal hollow.

b. With the available hand, the professional will take the patient's thigh (Figure 11). 


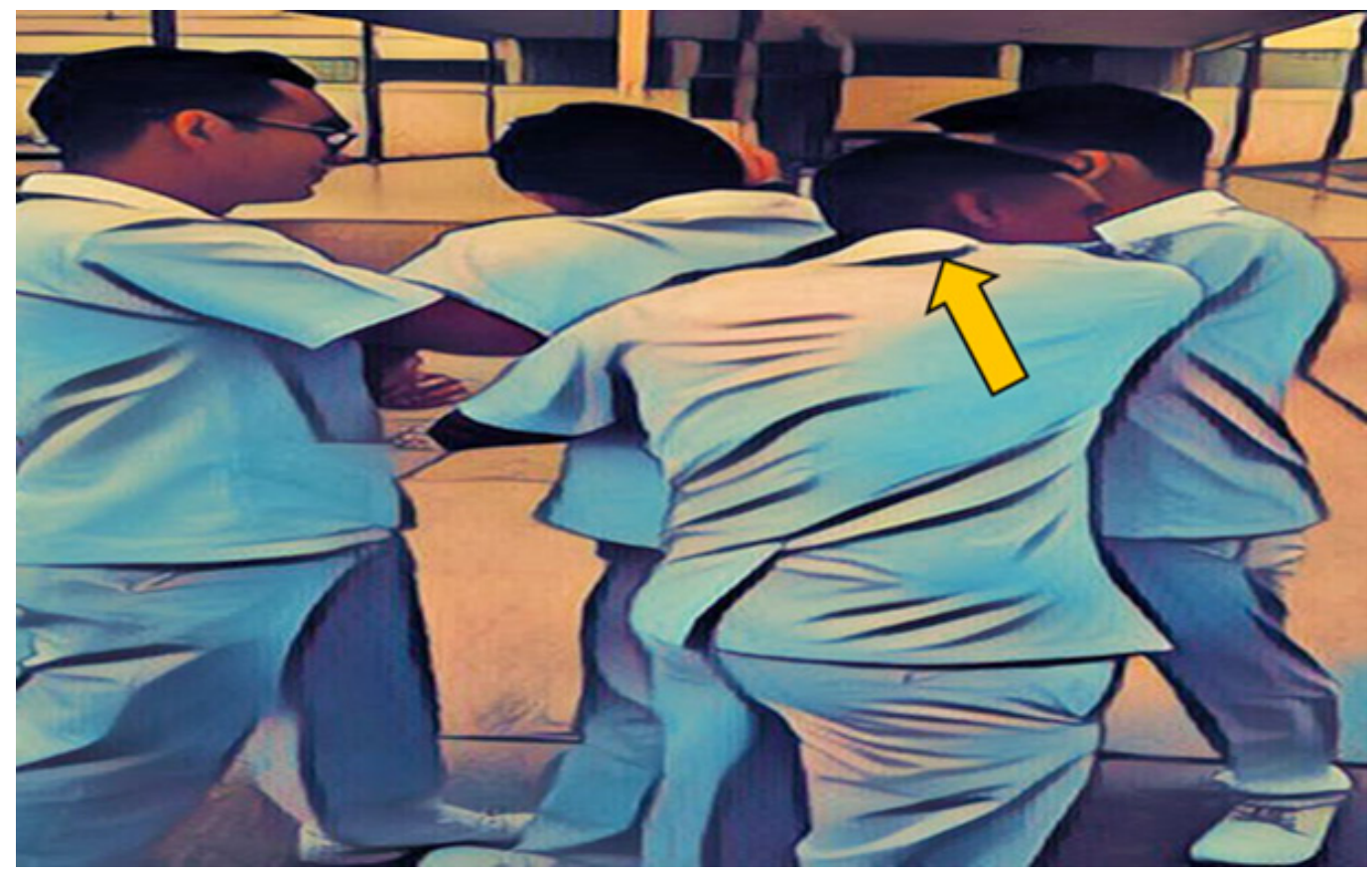

Figure 10:

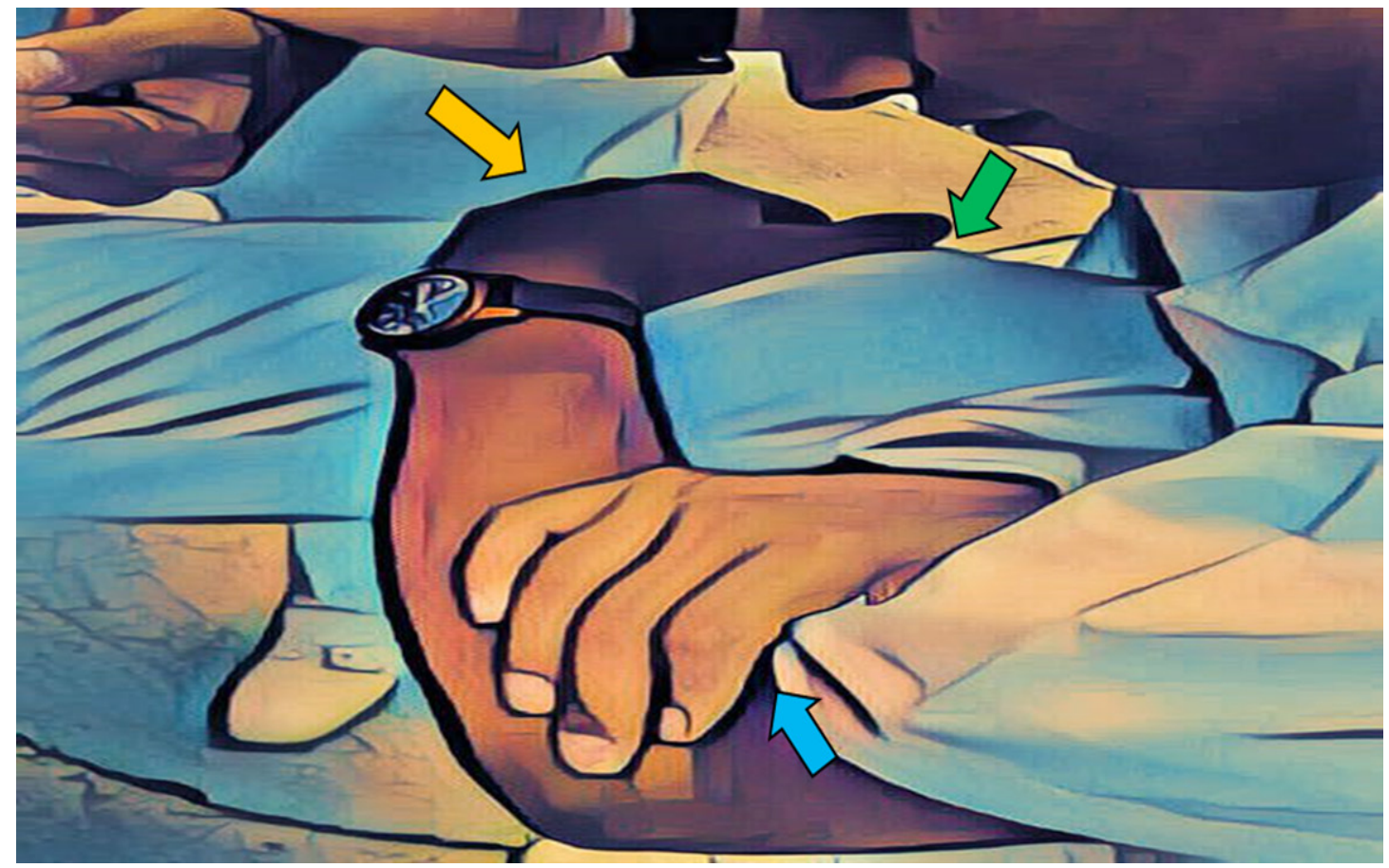

Figure 11:

c. The first hand will take the clench of the other hand of the professional (Figure 11), forming a "four resembling figure" with his arms, limiting and stabilizing the movements of the patient's knee joint.

7. Once the 4 limbs are secured, the Strategic Point Professional, who previously immobilized the thorax, will release the patient.

8. The professionals who are stabilizing the limbs of the patient, assist him by a gentle elevation, to help him/her to lay dorsal decubitus in the bed (Figure 12).

9. Once in bed, the manual immobilization of the 4 limbs should be maintained until the Strategic Point Professional, proceeds with the placement of the fastening material around the patient's wrists and ankles, permitting a free space between the skin of the latter and the fastening material, thus allowing proper circulation. 


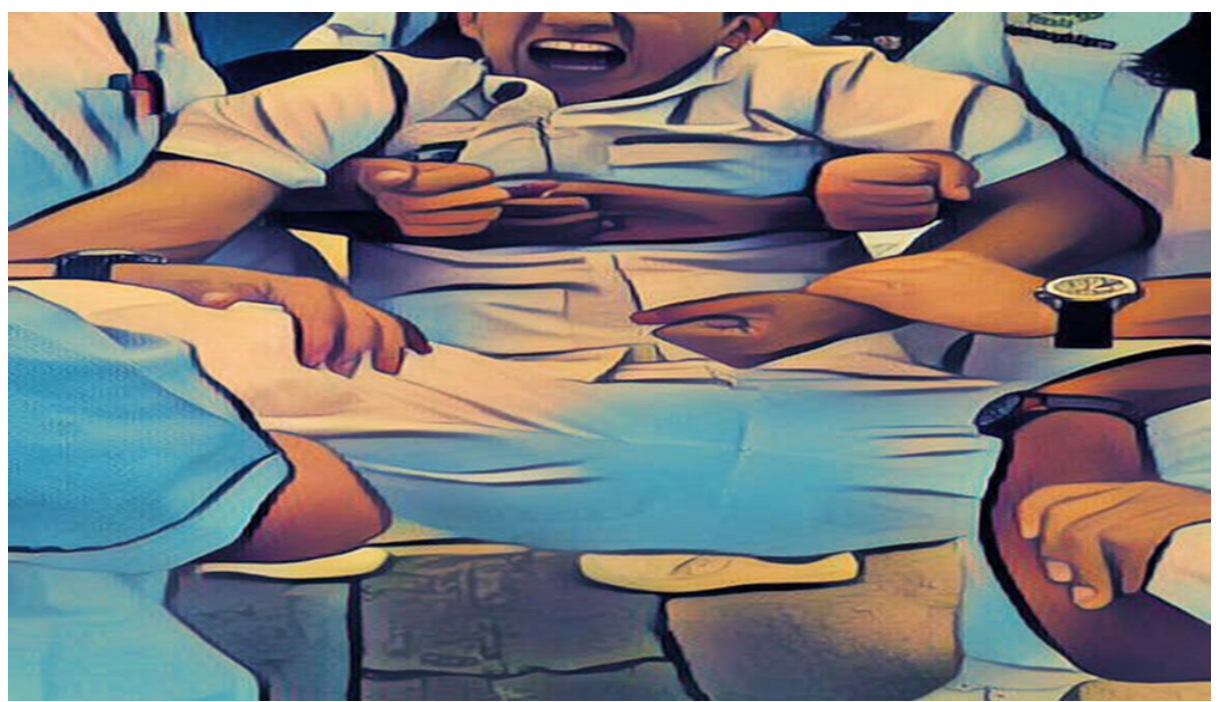

Figure 12:

\section{Nursing care management of a patient under physical containment}

Taking always on count that some negative outcomes have been described, it is essential to closely supervise the patient during the during physical containment including monitoring of general, clinical and safety variables of the patient each $15 \mathrm{~min}$, and maintained for as long as the patient is under physical containment. Once the HRS is resolved, the physical containment should be gradually removed while maintaining verbal containment, and explaining what behavior is expected from the patient, and with at least two professionals present18. The following list, summarizes the general nursing care management of a patient under physical containment:

1. Maintain a cordial and calmed atmosphere in the environment .

2. Guarantee at all times the conditions of comfort and safety of the patient.

3. Ensure that the fastening material do not hurt or lacerate the adjacent area of the patient's limb.

4. Avoid the use of bedding, sheets, blankets or any similar implement to make immobilization.

5. The bed must have a mattress that prevents the patient from getting hurt with the bed structure.

6. In the event that therapeutic support is needed in a wheelchair, it must have a padded seat and adequately secured to avoid movement, maintaining continuous patient supervision.

7. Review the pressure applied to the limb, verifying the circulation and capillary filling, as well as the mobility of the limb every 15 minutes.

8. Periodically and rotationally loosen the fasteners.

9. Keep track of vital signs, and their record in the clinical
10. Address the patient's hydration status.

11. Assess aspiration risk (solids and / or liquids).

12. Provide assistance in case the patient requires to satisfy an excretory need.

13. Avoid holding the position of fastening material directly on wounds or catheter insertion areas.

14. Monitor the general condition of the patient, attitude towards the containment, level of agitation, aggressiveness or anxiety.

15. Report immediately to medical/psychiatry staff, if there are any complications, or if harm risk situation is resolved, or if it is not responding as expected.

\section{Registration and informed consent}

As detention in a psychiatric context may lead to nonconsensual psychiatric treatment, falling under the scope of the Convention against Torture, where the fear and anxiety produced by indefinite detention, the infliction of forced medication or electroshock, the use of restraints and seclusion, the segregation from family and community are taken into account16, it is crucial to systematically and meticulously register the individual HRS for the patient and others, strictly adhering to the protocol and showing that there has been taken several actions to prevent any nonconsensual form treatment as the last available management option. The features of the patient in a HRS, the containment strategies, the failure to the deliver consensual management and appropriate engagement of the patient, the date and hours, the evolution and reevaluations of the HRS, the safety measures and monitoring for a patient on physical containment, the employed medications and the patient's response, should always to be recorded on the clinical file. A free informed consent should be the mainstay for any medical act, reflecting the respect for the patients' rights, with the only exception for emergency cases, the individual concerned he has been reliably shown to be of "unsound mind", so it is highly recommended to 
elaborate personalized informed consent formats, and a data record to track and monitor all of the procedures when handling a patient in a HRS.

\section{Conclusion}

To our knowledge this is the first complete therapeutic containment protocol in the management of HRS, that integrates the concepts of patient's safety and quality, promoting a clear standing regarding the human rights, torture prevention, and MI principles, integrating a comprehensive sequenced intervention, emphasizing the vantages of effectively delivered verbal containment, leaving the use of physical containment as the last alternative, and punctually describing a technique developed by our group, designed to address the safety for the patient and the staff while delivered. While is intended that this strategy could be a helpful and directive resource for MHF and healthcare services who manage patients in HRS, we acknowledge that the protocol and proposed algorithm could be too simplistic to accurately depict the complexities of the management in the diverse HRS, so it is subject for being modified and improved. We are also aware that it may be insufficiently highlighted the extent of human rights and discourage to use physical containment, besides the described procedure technique that will have to show its apparent safety, so we must emphasize that proper and frequent training is needed, along with the empowerment of the staff with the benefits of a well delivered verbal contention as the mainstay, that could effectively de-escalate the patient in most of HRS, preventing complications and bringing more satisfaction of the service.

\section{References}

1. Kilbourne AM, Beck K, Spaeth RB, Ramanuj P, Brien ORW, et al. (2018) Measuring and improving the quality of mental health care: A global perspective. World Psychiatry 17(1): 30-38.

2. Briner M, Manser T (2013) Clinical risk management in mental health: A qualitative study of main risks and related organizational management practices. BMC Health Serv Res 13: 44.

3. United Nations General Assembly (1991) Principles for the protection of persons with mental illness and the improvement of mental health care.

4. World Health Organization (2005) WHO resource book on mental health, human rights and legislation. World Health Organization, Geneva, Switzerland.

5. Ling S, Cleverley K, Perivolaris A (2015) Understanding mental health service patient experiences of restraint through debriefing: A Qualitative Analysis. Can J Psychiatry 60(9): 386-392.

6. Blanco SA, Valera RM, Lou AI, Baladón HL, Gibert K, et al. (2017) Inpatient costs of agitation and containment in a mental health catchment area. BMC Psychiatry 17(1): 212.

7. Huf G, Coutinho ES, Ferreira MA, Ferreira S, Mello F, et al. (2011) TRECSAVE: A randomized trial comparing mechanical restraints with use of seclusion for aggressive or violent seriously mentally ill people: study protocol for a randomized controlled trial. Trials 12: 180.

8. Beysard N, Yersin B, Carron PN (2018) Mechanical restraint in an emergency department: A consecutive series of 593 cases. Intern Emerg Med 13(4): 575-583.

9. Kalula, SZ, Petros SG (2016) Use of physical restraint in hospital patients: A descriptive study in a tertiary hospital in South Africa. Curationis 39(1): e1-e8.

10. Sailas E, Fenton M (2000) Seclusion and restraint for people with serious mental illnesses. Cochrane Database Syst Rev (2): CD001163.

11. (2015) National Collaborating Centre for Mental Health (UK) Violence and aggression: short-term management in mental health, health and community settings: Updated edition. British Psychological Society, London, United Kingdom.

12. Petrini C (2013) Ethical considerations for evaluating the issue of physical restraint in psychiatry. Ann Ist Super Sanità 49(3): 281-285.

13. Negroni AA (2017) On the concept of restraint in psychiatry. Eur J Psychiat 31(3): 99-104.

14. Muralidharan S, Fenton M (2006) Containment strategies for people with serious mental illness. Cochrane Database of Systematic Reviews.

15. Bowers L, James K, Quirk A, Simpson A, Stewart D, et al. (2015) Reducing conflict and containment rates on acute psychiatric wards: The safewards cluster randomised controlled trial. International journal of nursing studies 52(9): 1412-1422.

16. Méndez JE (2015) UN Human Rights Council, Report of the special rapporteur on torture and other cruel, inhuman or degrading treatment or punishment.

17. Knox DK, Holloman GH (2012) Use and avoidance of seclusion and restraint: Consensus statement of the American association for emergency psychiatry project Beta seclusion and restraint workgroup. West J Emerg Med 13(1): 35-40.

18. Vieta E, Garriga M, Cardete L, Bernardo M, Lombraña M, et al. (2017) Protocol for the management of psychiatric patients with psychomotor agitation. BMC Psychiatry 17: 328.

19. Fishkind A (2002) Calming agitation with words, not drugs: 10 commandments for safety. Current Psych 1(4).

20. Health Service Executive. Risk management in mental health services.

21. Stowell KR, Florence P, Harman HJ, Glick RL (2012) Psychiatric evaluation of the agitated patient: Consensus statement of the American association for emergency psychiatry project Beta psychiatric evaluation workgroup. West J Emerg Med 13(1): 11-6.

22. Garriga M, Pacchiarotti O, Kasper S, Zeller SL, Allen MH, et al. (2016) Assessment and management of agitation in psychiatry: expert consensus. World J Biol Psychiatry 17: 86-128.

23. Richmond JS, Berlin JS, Fishkind AB, Holloman GH, Zeller SL, et al. (2012) Verbal De-escalation of the Agitated Patient: Consensus Statement of the American Association for Emergency Psychiatry Project BETA Deescalation Workgroup. West J Emerg Med 13(1): 17-25.

24. Wilson MP, Pepper D, Currier GW, Holloman GH, Feifel D (2012) The psychopharmacology of agitation: consensus statement of the American association for emergency psychiatry project Beta psychopharmacology workgroup. West J Emerg Med 13(1): 26-34.

For possible submissions Click below: 